\title{
Efficient hedging under ambiguity in continuous time
}

\section{Ludovic Tangpi}

Received: 13 March 2019 / Accepted: 13 August 2020 / Published online: 28 August 2020 (C) The Author(s). 2020 Open Access This article is licensed under a Creative Commons Attribution 4.0 International License, which permits use, sharing, adaptation, distribution and reproduction in any medium or format, as long as you give appropriate credit to the original author(s) and the source, provide a link to the Creative Commons licence, and indicate if changes were made. The images or other third party material in this article are included in the article's Creative Commons licence, unless indicated otherwise in a credit line to the material. If material is not included in the article's Creative Commons licence and your intended use is not permitted by statutory regulation or exceeds the permitted use, you will need to obtain permission directly from the copyright holder. To view a copy of this licence, visit http://creativecommons.org/licenses/by/4.0/.

\begin{abstract}
It is well known that the minimal superhedging price of a contingent claim is too high for practical use. In a continuous-time model uncertainty framework, we consider a relaxed hedging criterion based on acceptable shortfall risks. Combining existing aggregation and convex dual representation theorems, we derive duality results for the minimal price on the set of upper semicontinuous discounted claims.
\end{abstract}

Keywords Superhedging · model ambiguity - Acceptance set · Risk measure · Optimized certainty equivalent $\cdot$ Volatility uncertainty

MSC 2010 91B30 $\cdot 91 \mathrm{G} 80 \cdot 60 \mathrm{H} 30 \cdot 60 \mathrm{G} 48$

\section{Introduction}

In this paper, we are concerned with convex duality for the minimal superhedging problem with non-zero shortfall risk in continuous time. In a financial market with underlying $S$, the minimal superhedging price $\phi(X)$ of a discounted contingent claim $X$ is the smallest cost $m$ needed to form a superhedging portfolio. That is, to find an admissible strategy $Z$ such that

$$
m+(Z \cdot S)_{T} \geq X
$$

\footnotetext{
L. Tangpi $(\square)$

Princeton University, Department of Operations Research and Financial Engineering, Princeton 08540, NJ, USA

e-mail: ludovic.tangpi@princeton.edu
} 
where $(Z \cdot S)_{T}$ is the total gain up to time $T \in(0, \infty)$ from trading $S$. A classical result at the heart of mathematical finance gives conditions guaranteeing a pricinghedging duality, ensuring, for instance, that $\phi(X)$ is the largest non-arbitrage price of $X$, see, for example, Delbaen and Schachermayer (1994) for details, and Kramkov and Schachermayer (1999) for applications to portfolio optimization.

In the presence of model ambiguity, that is, when the negligible events do not stem from a single measure, the pricing-hedging duality has attracted a sustained of attention. Notably under models with volatility uncertainty, such duality results have been derived, for instance, by Peng (2010), Denis and Martini (2006), and Soner et al. (2013) and Soner et al. (2011a) for contingent claims that are (versions of) continuous random variables. A crucial step to derive most of these results is to prove a dynamic programming principle. This requires the ("dynamic version" of the functional) $\phi$ to be time-consistent. Neufeld and Nutz (2013) have extended these representations (and the dynamic programming principles) to measurable claims using the theory of analytic sets. In the model-free framework, that is, when no probabilistic assumption is made, superhedging duality results include those in (Acciaio et al. 2016; Beiglböck et al. 2013; Cheridito et al. 2017; Burzoni et al. 2017; Burzoni 2016) in discrete time and (Dolinsky and Soner 2014; Hou and Obłój 2018; Bartl et al. 2019; Bartl et al. 2020) in continuous time.

It is well-known that the minimal superhedging price is too high for practical use, and even higher under model uncertainty. This motivated the notion of quantilehedging introduced by Föllmer and Leukert (1999) and Föllmer and Leukert (2000) and further developed into risk-based approaches by Arai (2010) and Rudloff (2007); and into no-good deal based valuations by Bion-Nadal and Di Nunno (2013). We also refer to Becherer and Kentia (2017) for the analysis of robust no-good deals. More precisely, this consists in substituting the (strict) superhedging requirement (1) by the relaxed condition

$$
m+(Z \cdot S)_{T}-X \in \mathcal{A}
$$

where $\mathcal{A}$ is the acceptance set of a convex monetary risk measure, or a set of acceptable discounted financial positions. Adjusting the set $\mathcal{A}$ allows to change the level of risk aversion. Under model uncertainty, superhedging dualities in such a situation have been investigated by Cheridito et al. (2017) in the discrete-time model-free framework.

The goal of the present paper is to investigate the continuous-time case when a set $\mathcal{P}$ of possible reference measures on the canonical space $C\left([0, T], \mathbb{R}^{d}\right)$ is fixed. Notice that if the risk measure with acceptance set $\mathcal{A}$ is not time-consistent, then the resulting superhedging functional is not necessarily time-consistent anymore (for instance, think of the trivial case where $S=0$ ). This renders the dynamic programming approach prevalent in the literature harder to apply. The proposed argument is based on results by Cheridito et al. (2015) which give conditions under which a continuity from below condition (also known as the Fatou property) yields a representation of convex monotone functions. More precisely, we show that a suitable sequential closedness of the acceptance set $\mathcal{A}$ carries over to the sublevel sets of the superhedging functional, guaranteeing enough regularity to derive a convex dual representation; see Theorem 1 for a precise statement. This will require the use of aggregation results developed by Soner et al. (2011b). As an application, the case where the shortfall risk 
is quantified by a robust optimized certainty equivalent is systematically studied. To give a taste of our results, consider the set of reference measures

$$
\mathcal{P}:=\left\{P: \underline{a} \leq d\langle S\rangle_{t} / d t \leq \bar{a}: P \otimes d t-\text { a.s. }\right\}
$$

with $S$ being the canonical process on the space of continuous paths, $\underline{a}$ and $\bar{a}$ being two symmetric, positive definite deterministic matrices. This is an important class of reference measures as it pertains to volatility uncertainty, and is also linked to Peng's

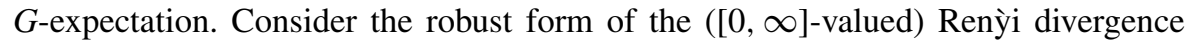
given by

$$
\rho^{*}(Q):=\inf _{P \in \mathcal{P}} \frac{1}{q} E_{P}\left[\left(\frac{d Q}{d P}\right)^{q}\right] \text { for some } q>1
$$

and the associated risk measure (see Bartl et al. (2020)) defined as

$$
\rho(X):=\sup _{Q}\left(E_{Q}[-X]-\rho^{*}(Q)\right)
$$

with the supremum being over all regular probability measures, with the understanding $\frac{d Q}{d P}=+\infty$ if $Q$ is not absolutely continuous with respect to $P$. We will show, see Theorem 2 and Example 1, that if a contingent claim $X$ is bounded and upper semicontinuous (on the path space equipped with the supremum norm), then it holds

$$
\begin{aligned}
& \inf \left\{m \in \mathbb{R}: \rho\left(m+\int_{0}^{T} Z_{u} d S_{u}-X\right) \leq 0 P \text {-a.s. for all } P \in \mathcal{P}\right\} \\
& \quad=\sup _{Q}\left(E_{Q}[X]-\rho^{*}(Q)\right),
\end{aligned}
$$

where the supremum is taken over martingale measures for $S$ with densities with respect to $P \in \mathcal{P}$ having finite $q$ moments. Recall that the risk measure $\rho$ is not time-consistent, see (Kupper and Schachermayer 2009).

In the next section, we describe the probabilistic setting and the main results of the paper. Namely, a convex dual representation for the superhedging functional for upper semicontinuous claims when the shortfall risk is quantified by a risk measure whose acceptance set satisfies some integrability property. As an example, the case of robust optimized certainty equivalent is studied in detail, since this class of risk measures includes a large number of examples, see, for instance, (Bartl et al. 2020; Ben-Tal and Taboulle 2007). All the proofs are given in section 3, and an appendix contains some technical concepts from (Soner et al. 2011b).

\section{Setting and main results}

\subsection{Probabilistic setting}

The findings of this work rely on representation results of Cheridito et al. (2015) and the aggregation results of Soner et al. (2011b) from which we borrow the probabilistic setting. More precisely, fix $T \in(0, \infty), d \in \mathbb{N} \backslash\{0\}$ and let $\Omega$ be the canonical space of $\mathbb{R}^{d}$-valued continuous paths on $[0, T]$ with $\omega_{0}=0$. Let $P_{0}$ be the Wiener measure on $\Omega$ and $S$ the canonical process, with natural filtration $\mathbb{F}^{S}=\left(\mathcal{F}_{t}^{S}\right)_{t \in[0, T]}$. 
By Karandikar (1983), there exists an $\mathbb{F}^{S}$-adapted, continuous process $\langle S\rangle$, such that $\langle S\rangle_{t}=\langle S\rangle_{t}^{Q} Q$-a.s. (almost surely) for all $t \in[0, T]$, and every local martingale measure $Q$ of $S$, where $\langle S\rangle^{Q}$ denotes the $Q$-quadratic variation of $S$. Let $\hat{a}$ be the density of the quadratic variation $\langle S\rangle$ given by

$$
\hat{a}_{t}:=\limsup _{\varepsilon \downarrow 0} \frac{1}{\varepsilon}\left(\langle S\rangle_{t}-\langle S\rangle_{t-\varepsilon}\right) .
$$

We denote by $\mathcal{M}(S)$ the set of all local martingale measures $P$ such that $P$-a.s., $\langle S\rangle_{t}$ is absolutely continuous in $t$ and $\hat{a}$ is valued in the set $\mathbb{S}_{d}^{>0}$ of symmetric positive definite matrices. For every $P \in \mathcal{M}(S)$ and every integrable $\mathbb{F}^{S}$-progressively measurable process $a$ taking values in $\mathbb{S}_{d}^{>0}$, and such that $a=\hat{a} P$-a.s. (such process $a$ is called diffusion coefficient), $P$ is a weak solution of the SDE

$$
d Y_{t}=a^{1 / 2}(Y .) d S_{t} \quad P_{0} \text {-a.s. }
$$

with initial value $P\left(S_{0}=0\right)=1$. In particular, $S$ is a $P$-local martingale. By (Karatzas and Shreve 2004), the SDE (3) admits a unique weak solution for every bounded process with values in $\mathbb{S}_{d}^{>0}$. Let $A_{0}$ be a generating class of diffusion coefficients (see Definition 1) such that every $a \in A_{0}$ is bounded and $P^{a}$ satisfies the martingale representation property. Further, let $A$ be a separable class of diffusion coefficients generated by $A_{0}$, see Definition 2 , and put

$$
\mathcal{P}:=\left\{P^{a}: a \in A\right\} .
$$

We consider the set $\mathcal{P}$ as the set of reference probability measures. Intuitively, the set $\mathcal{P}$ is the set of models for different volatilities $a \in A$. For the sake of flexibility, we allow the choice of $a$ to be such that (3) has only a weak solution. One could also consider the "strong formulation" (as we did in the introduction), where $P^{a}:=$ $P_{0} \circ\left(Y^{a}\right)^{-1}$ with $Y_{t}^{a}:=\int_{0}^{t} a_{s}^{1 / 2} d S_{s}$ for some process $a$. For every $P \in \mathcal{P}$, let $\mathbb{F}^{P}:=$ $\left(\mathcal{F}_{t}^{P}\right)_{t \in[0, T]}$ be the $P$-completion of the right-continuous version of the filtration $\mathbb{F}^{S}$, and denote by $\mathbb{F}:=\left(\mathcal{F}_{t}\right)_{t \in[0, T]}$ the filtration given by $\mathcal{F}_{t}:=\bigcap_{P \in \mathcal{P}}\left(\mathcal{F}_{t}^{P} \vee \mathcal{N}^{\mathcal{P}}\right)$, where $\mathcal{N}^{\mathcal{P}}$ is the collection of $P$-null sets for all $P \in \mathcal{P}$.

Let $L^{0}(\mathcal{P})$ be the space of $\mathcal{F}_{T}$-measurable random variables which are identified if they agree $\mathcal{P}$-q.s. ${ }^{1}$ and, given $p \in[1, \infty)$, we denote by $L^{p}(\mathcal{P})$ the space of random variables $X \in L^{0}(\mathcal{P})$ such that $E_{P}\left[|X|^{p}\right]<\infty$ for all $P \in \mathcal{P}$. Further, let $L^{\infty}(\mathcal{P})$ be the subspace of $L^{p}(\mathcal{P})$ equipped with the norm $\|X\|_{\infty}:=\inf \{m>0$ : $\left.\sup _{P \in \mathcal{P}} P(|X|>m)=0\right\}$.

\subsection{Main results}

For every progressively measurable $\mathbb{R}^{d}$-valued processes $Z$ such that $\int_{0}^{T}\left|Z_{t}\right|^{2} d\langle S\rangle_{t}<\infty$, we denote by $\int Z d S$ the usual Itô's integral which implicitly depends on $P \in \mathcal{P}$ and by $M^{Z}$ the $\mathcal{P}$-q.s. unique $\mathbb{F}$-progressively measurable process such that $M^{Z}=\int Z d S P$-a.s. for all $P \in \mathcal{P}$ (see (Soner et al. 2011b, Theorem 6.4)).

\footnotetext{
${ }^{1}$ Hereby $\mathcal{P}$-q.s. means $P$-a.s. for every $P \in \mathcal{P}$. Unless otherwise stated, all equalities and inequalities between random variables will be understood in this sense.
} 
This defines a $\mathcal{P}$-local martingale, that is, a $P$-local martingale under each $P \in \mathcal{P}$. The (admissible) gains and losses from trading in the financial market modeled by $S$ are given by the set

$$
G:=\left\{M_{T}^{Z}: \int_{0}^{t} Z_{u} d S_{u} \geq-c \text { for all } t \in[0, T], \text { for some } c>0\right\}
$$

and the minimal superhedging cost $\phi(X)$ of a contingent claim $X \in L^{0}(\mathcal{P})$, is given by

$$
\phi(X):=\inf \{m \in \mathbb{R}: m+Y \geq X \text { for some } Y \in G\} .
$$

Fix a non-empty, convex set $\mathcal{A} \subseteq L^{0}(\mathcal{P})$ and containing $L_{+}^{0}(\mathcal{P})$ assumed to be monotone ${ }^{2}$. The functional $\psi$ given by

$$
\psi(X):=\inf \{m \in \mathbb{R}: m+Y-X \in \mathcal{A} \text { for some } Y \in G\}
$$

defines the minimal cost to be paid to construct a portfolio with a shortfall that lies in the set $\mathcal{A}$ but that may fail to superhedge the claim $X$ (in the $\mathcal{P}$-q.s. sense), with the convention $\inf \emptyset:=+\infty$. Our aim is to derive the dual representation of the functional $\psi$.

Let $C_{b}$ and $U_{b}$ be the space of bounded continuous functions and bounded upper semicontinuous functions on $\Omega$, and $C_{b}(\mathcal{P})$ and $U_{b}(\mathcal{P})$ the set of elements of $L^{\infty}(\mathcal{P})$ with a $\mathcal{P}$-q.s. version in $C_{b}$ and $U_{b}$, respectively. Put

$$
\psi^{*}(Q):=\sup _{X \in C_{b}}\left(E_{Q}[X]-\psi(X)\right) \quad \text { and } \quad \phi^{*}(Q):=\sup _{X \in C_{b}}\left(E_{Q}[X]-\phi(X)\right)
$$

and denote by $\rho_{\mathcal{A}}$ and $\rho_{\mathcal{A}}^{*}$, respectively, the risk measure associated to $\mathcal{A}$ and its conjugate, that is,

$$
\rho_{\mathcal{A}}(X):=\inf \{m: m+X \in \mathcal{A}\} \quad \text { and } \quad \rho_{\mathcal{A}}^{*}(Q):=\sup _{X \in C_{b}}\left(E_{Q}[X]-\rho_{\mathcal{A}}(X)\right) .
$$

The convex conjugates $\psi^{*}, \phi^{*}$, and $\rho_{\mathcal{A}}^{*}$ are convex and lower semicontinuous functions and play a key role in the statement and proofs of our dual representation results. In the course of the proofs of Theorems 1 and 2 we will show that $\psi^{*}(Q)=$ $\phi^{*}(Q)+\rho^{*}(-Q)$, (where we use the informal notation $E_{-Q}[X]:=E_{Q}[-X]$ ) and that $\phi^{*}(Q)$ is zero for martingale measures and infinity otherwise.

Denote by $\mathrm{ca}_{1}^{+}$the set of Borel probability measures on $\Omega, c a_{1}^{+}(\tilde{\Omega})$ its subset containing probability measures with support included in $\tilde{\Omega}:=\operatorname{supp}(\mathcal{P})$, the $\operatorname{support}^{3}$ of $\mathcal{P}$, and by $\mathcal{M}_{\mathcal{P}}^{\mathcal{A}}(S)$ the set of probability measures $Q \in c a_{1}^{+}(\widetilde{\Omega})$ such that $S$ is a $Q$-local martingale and it holds $\rho_{\mathcal{A}}^{*}(-Q)<\infty$.

Theorem 1 Assume that the set $\mathcal{A}^{-}:=\left\{A^{-}: A \in \mathcal{A}\right\}$ is $\mathcal{P}$-uniformly integrable $^{4}$ the sublevel sets $\left\{Q \in c a_{1}^{+}: \rho_{\mathcal{A}}^{*}(-Q) \leq c\right\}, c \geq 0$ are weakly compact

\footnotetext{
${ }^{2}$ In the sense that for every $X, X^{\prime} \in L^{1}(\mathcal{P})$ with $X \geq X^{\prime}$ and $X^{\prime} \in \mathcal{A}$, we have $X \in \mathcal{A}$.

${ }^{3}$ Here, $\operatorname{supp}(\mathcal{P})$ is the unique closed set $\widetilde{\Omega}$ for which $P\left(\widetilde{\Omega}^{c}\right)=0$ for all $P \in \mathcal{P}$ and $P(\widetilde{\Omega} \cap O)>0$ for some $P \in \mathcal{P}$ whenever $O$ is open and $\widetilde{\Omega} \cap O \neq \emptyset$. It can be checked that $\operatorname{supp}(\mathcal{P})=\cup_{P \in \mathcal{P}} \operatorname{supp}(P)$, where $\operatorname{supp}(P)$ exists, since $P$ is a regular measure, see (Aliprantis and Border 2006, Theorem 12.14).

${ }^{4}$ That is, it is $P$-uniformly integrable for each $P \in \mathcal{P}$; and $A^{-}:=\max (0,-A)$.
} 
and $\liminf _{n \rightarrow \infty} A^{n} \in \mathcal{A}$ for every sequence $\left(A^{n}\right)$ in $\mathcal{A}$ that is bounded in $L^{1}(\mathcal{P})$. Then, if $\psi(0)>-\infty$, the functional $\psi$ is real-valued on $L^{\infty}(\mathcal{P})$ and satisfies the representation ${ }^{5}$

$$
\psi(X)=\sup _{Q \in \mathcal{M}_{\mathcal{P}}^{\mathcal{A}}(S)}\left(E_{Q}[X]-\rho_{\mathcal{A}}^{*}(-Q)\right), \quad X \in C_{b}(\mathcal{P}) .
$$

Moreover, if

$$
\psi^{*}(Q)=\sup _{X \in U_{b}}\left(E_{Q}[X]-\psi(X)\right)
$$

then one has that

$$
\psi(X)=\sup _{Q \in \mathcal{M}_{\mathcal{P}}^{\mathcal{A}}(S)}\left(E_{Q}[X]-\rho_{\mathcal{A}}^{*}(-Q)\right), \quad X \in U_{b}(\mathcal{P}) .
$$

The proof of this result is given in subsection 3.1. This result is close in spirit to the so-called no-good deal bounds derived by (Becherer and Kentia 2017) using second order backward stochastic differential equations.

Remark 1 Since $G$ is convex, the convexity and monotonicity conditions on $\mathcal{A}$ ensure that $\psi$ is increasing and convex on the vector space $L^{1}(\mathcal{P})$. When $\mathcal{A}=$ $L_{+}^{0}(\mathcal{P})$, then $\psi$ reduces to the superhedging function $\phi$. In Theorem 1 , assuming $\psi(0)>-\infty$ can be seen as a market viability condition, it is satisfied, for instance, if $\mathbb{R}_{+} \cap(G-\mathcal{A})=\{0\}$, compare (Cheridito et al. 2017), or if there is a probability measure $Q$ such that $E_{Q}[A] \geq 0$ for all $A \in \mathcal{A}$ and $E_{Q}[Y] \leq 0$ for all $Y \in G$. Moreover, the uniform integrability condition on $\mathcal{A}^{-}$prevents, in particular, that $\rho_{\mathcal{A}}$ attains the value $-\infty$ which is undesirable for a risk measure. Furthermore, assuming that $\liminf _{n \rightarrow \infty} X^{n} \in \mathcal{A}$ for every sequence $\left(X^{n}\right)$ in $\mathcal{A}$ that is bounded in $L^{1}(\mathcal{P})$ can be seen as a version of the Fatou's property for risk measures on $L^{p}$-spaces, see, for instance, (Kaina and Rüschendorf 2009).

A particularly interesting case arises when $\mathcal{A}$ is the acceptance set of a robust optimized certainty equivalent. More precisely, let $l: \mathbb{R} \rightarrow \mathbb{R}$ be a loss function satisfying the usual assumptions

$l$ is convex, increasing, bounded from below, and $l(0)=0, l^{*}(1)=0$, and $l(x)>x$ for $|x|$ large enough, $\}$

where $l^{*}$ denotes the convex conjugate of $l$ defined as

$$
l^{*}(y):=\sup _{x \in \mathbb{R}}(x y-l(x))
$$

for $y \in \mathbb{R}$ and $l^{*}(+\infty):=+\infty$. The functional $\rho: L^{1}(\mathcal{P}) \rightarrow(-\infty, \infty]$ defined by

$$
\rho(X):=\inf _{m \in \mathbb{R}} \sup _{P \in \mathcal{P}}\left(E_{P}[l(m-X)]-m\right)
$$

\footnotetext{
${ }^{5} \operatorname{In}(6), E_{Q}[X]$ is understood as $E_{Q}\left[X^{\prime}\right]$, for any $X^{\prime} \in C_{b}$ with $X=X^{\prime} \mathcal{P}$-q.s. This expectation is uniquely defined, see (Tangpi 2015, Lemma 4.5.1).
} 
is the analogue, in the context of model ambiguity, of the optimized certainty equivalent risk measure introduced by (Ben-Tal and Taboulle 2007). It satisfies

$$
\rho(X)=\sup _{Q \in c a_{1}^{+}}\left(E_{Q}[-X]-\inf _{P \in \mathcal{P}} E_{P}\left[l^{*}\left(\frac{d Q}{d P}\right)\right]\right), \quad X \in L^{\infty}(\mathcal{P}),
$$

where $d Q / d P:=\infty$ if $Q$ is not absolutely continuous w.r.t. $P$ and with the understanding $E_{P}[Z]:=\infty$ whenever $E_{P}\left[Z^{+}\right]=\infty$, see (Bartl et al. 2020) for details. Let us consider the acceptance set

$$
\mathcal{A}:=\left\{X \in L^{1}(\mathcal{P}): \rho(X) \leq 0\right\}
$$

and denote by $\mathcal{M}_{\mathcal{P}}^{l}(S)$ the set of probability measures $Q \in c a_{1}^{+}(\tilde{\Omega})$ such that $S$ is a $Q$-local martingale and it holds $\inf _{P \in \mathcal{P}} E_{P}\left[l^{*}(d Q / d P)\right]<\infty$.

Theorem 2 Assuming that $l$ satisfies $(C I B)$, there exist $a>0, b \in \mathbb{R}$, and $p>2$ such that $l(x) \geq a|x|^{p}+b$ and $\psi(0)>-\infty$. If $\mathcal{P}$ is weakly compact, then it holds

$$
\psi(X)=\sup _{Q \in \mathcal{M}_{\mathcal{P}}^{l}(S)}\left(E_{Q}[X]-\inf _{P \in \mathcal{P}} E_{P}\left[l^{*}(d Q / d P)\right]\right), \quad X \in U_{b}(\mathcal{P})
$$

The proof of this result is given in subsection 3.2.

Example 1 Let $\underline{a}, \bar{a}$ be two matrices with $0<\underline{a} \leq \bar{a}$, and denote by $A_{0}$ the set of (deterministic) functions on $[0, T]$ valued in $\mathbb{S}_{d}^{>0}$ and such that $\underline{a} \leq a_{t} \leq \bar{a}$ for all $t \in[0, T]$. By (Soner et al. 2011b, Example 4.9), $A_{0}$ is a generating class of diffusion coefficients and it clearly generates itself (in the sense of Definition 2). Put

$$
\mathcal{P}:=\left\{P: d\langle S\rangle_{t}^{P} / d t \in A_{0}-P \otimes d t \text { a.s. }\right\}
$$

It follows from (Bion-Nadal and Kervarec 2012, Proposition 6.2) that the set $\mathcal{P}$ is compact in the weak topology. In this setting, taking for instance $l(x)=\left(x^{+}\right)^{p} / p$ with $p>2$, it follows from Theorem 2 that $\psi$ satisfies the representation

$$
\psi(X)=\sup _{Q \in \mathcal{M}_{\mathcal{P}}^{l}(S)}\left(E_{Q}[X]-\inf _{P \in \mathcal{P}} \frac{1}{q} E_{P}\left[\left(\frac{d Q}{d P}\right)^{q}\right]\right), \quad X \in U_{b}(\mathcal{P})
$$

with $q$ the Hölder conjugate of $p$.

Theorem 2 also applies to the entropic risk measure (which is time-consistent) when we let $l(x)=e^{x}-1$. In this case, it holds $l^{*}(z)=z \log (z)-z+1$ and

$$
\psi(X)=\sup _{Q \in \mathcal{M}_{\mathcal{P}}^{l}(S)}\left(E_{Q}[X]-\inf _{P \in \mathcal{P}} E_{P}\left[\frac{d Q}{d P} \log \left(\frac{d Q}{d P}\right)\right]\right), \quad X \in U_{b}(\mathcal{P})
$$




\section{Proofs}

\subsection{Proof of theorem 1}

For each $c>0$, consider the set $G^{c}:=\left\{M_{T}^{Z}: \int_{0}^{t} Z_{u} d S_{u} \geq-c\right.$ for all $\left.t \in[0, T]\right\}$ and the functional

$$
\psi^{c}(X):=\inf \left\{m \in \mathbb{R}: m+Y-X \in \mathcal{A} \text { for some } Y \in G^{c}\right\} .
$$

Recall that the inf-convolution $\rho_{1} \square \rho_{2}$ of two functions $\rho_{1}$ and $\rho_{2}$ on $L^{1}(\mathcal{P})$ is defined by

$$
\rho_{1} \square \rho_{2}(X):=\inf _{Y \in L^{1}(\mathcal{P})}\left(\rho_{1}(X-Y)+\rho_{2}(Y)\right) .
$$

The minimal cost $\psi^{c}$ can be written as an inf-convolution:

Lemma 1 For every $X \in L^{\infty}(\mathcal{P})$, the minimal cost $\psi^{c}$ satisfies $\psi^{c}(X)=$ $\rho_{\mathcal{A}} \square \bar{\phi}^{c}(-X)$, whereby $\bar{\phi}^{c}(\cdot):=\phi^{c}(-\cdot)$ and $\phi^{c}(X):=\inf \{m \in \mathbb{R}: m+Y-X \geq$ 0 for some $\left.Y \in G^{c}\right\}$.

Proof Let $X \in L^{\infty}(\mathcal{P}), \varepsilon>0$ and $Y \in G^{c}$. There is an $m \in \mathbb{R}$ such that $\rho_{\mathcal{A}}(Y-$ $X) \geq m-\varepsilon$ and $m+Y-X \in \mathcal{A}$. Hence, $\psi^{c}(X) \leq m \leq \rho_{\mathcal{A}}(Y-X)+\varepsilon$. This implies that $\psi^{c}(X) \leq \inf _{Y \in G^{c}} \rho_{\mathcal{A}}(Y-X)$. On the other hand, if $\inf _{Y \in G^{c}} \rho_{\mathcal{A}}(Y-X)=-\infty$, the previous inequality is an equality. If $\inf _{Y \in G^{c}} \rho_{\mathcal{A}}(Y-X)>-\infty$, let $m \in \mathbb{R}$ be such that $m<\inf _{Y \in G^{c}} \rho_{\mathcal{A}}(Y-X)$. Then it holds $m \leq \psi^{c}(X)$ because, if not, there would exist $Y \in G^{c}$ such that $m+Y-X \in \mathcal{A}$. That is, $m \geq \rho_{\mathcal{A}}(Y-X)$. Therefore,

$$
\psi^{c}(X)=\inf _{Y \in G^{c}} \rho_{\mathcal{A}}(Y-X) .
$$

In particular, denoting by $\bar{\phi}^{c}$ the functional $\bar{\phi}^{c}(X):=\inf \left\{m: m+X \in L_{+}^{0}(\mathcal{P})-G^{c}\right\}$, we have

$$
\psi^{c}(X) \geq \inf _{Y \in L^{1}(\mathcal{P})}\left(\rho_{\mathcal{A}}(-Y-X)+\bar{\phi}^{c}(Y)\right)
$$

and if we take $m>\inf _{Y \in L^{1}(\mathcal{P})}\left(\rho_{\mathcal{A}}(-Y-X)+\bar{\phi}^{c}(Y)\right)$, then for every $\varepsilon>0$ there exists $Y^{\prime} \in L^{1}(\mathcal{P})$ such that $m>\rho_{\mathcal{A}}\left(-Y^{\prime}-X\right)+\bar{\phi}^{c}\left(Y^{\prime}\right)-\varepsilon$. Thus, using the definition of $\bar{\phi}^{c}$, one can find $Y \in G^{c}$ such that $\bar{\phi}^{c}\left(Y^{\prime}\right)+Y \geq-Y^{\prime}-\varepsilon$. Since $\rho_{\mathcal{A}}$ is decreasing and translation invariant, this yields $m>\rho_{\mathcal{A}}(Y-X)-2 \varepsilon$, thus $m \geq \inf _{Y \in G^{c}} \rho_{\mathcal{A}}(Y-X)$ so that

$$
\psi^{c}(X)=\inf _{Y \in L^{1}(\mathcal{P})}\left(\rho_{\mathcal{A}}(-Y-X)+\bar{\phi}^{c}(Y)\right)=\rho_{\mathcal{A}} \square \bar{\phi}^{c}(-X) .
$$

Proposition 1 Under the conditions of Theorem 1, it holds:

(i) For every claim $X \in L^{0}(\mathcal{P})$ with $X^{-} \in L^{1}(\mathcal{P})$ and $\psi^{c}(X)<\infty$, there exists an optimal $\bar{Y} \in G^{c}$ such that $\psi^{c}(X)+\bar{Y}-X \in \mathcal{A}$. 
(ii) The functional $\psi^{c}$ is real-valued on $L^{\infty}(\mathcal{P})$ and satisfies

$$
\begin{array}{ll}
\psi^{c}(X)=\sup _{Q \in \mathcal{M}_{\mathcal{P}}^{\mathcal{A}}}\left(E_{Q}[X]-\psi^{c, *}(Q)\right), \quad X \in C_{b}(\mathcal{P}) \\
\psi^{c}(X) \leq \sup _{Q \in \mathcal{M}_{\mathcal{P}}^{\mathcal{A}}}\left(E_{Q}[X]-\psi^{c, *}(Q)\right), \quad X \in U_{b}(\mathcal{P})
\end{array}
$$

with $\mathcal{M}_{\mathcal{P}}^{\mathcal{A}}$ the set of probability measures $Q \in \operatorname{ca} a_{1}^{+}(\tilde{\Omega})$ such that $\rho_{\mathcal{A}}^{*}(-Q)<$ $\infty$. Moreover, it holds that

$$
\psi^{c, *}(Q)=\phi^{c, *}(Q)+\rho_{\mathcal{A}}^{*}(-Q),
$$

for all $Q \in c a_{1}^{+}(\tilde{\Omega})$.

Proof (i) Existence: We start by fixing a random variable $X \in L^{0}(\mathcal{P})$ such that $X^{-} \in L^{1}(\mathcal{P})$ and $\psi^{c}(X)<\infty$. There is a decreasing sequence of real numbers $\left(m^{n}\right)$ converging to $\psi^{c}(X)$ which is such that for all $n \in \mathbb{N}$ there exists $Y^{n} \in G^{c}$ satisfying

$$
m^{n}+Y^{n}-X \in \mathcal{A}, \quad \text { with } \quad Y^{n}:=\int_{0}^{T} Z_{u}^{n} d S_{u} .
$$

Let $M^{Z^{n}}$ be the unique process such that $M_{t}^{Z^{n}}=\int_{0}^{t} Z_{u}^{n} d S_{u} \mathcal{P}$-q.s. It can be checked that $M^{Z^{n}}$ is a $P$-supermartingale for each $n \in \mathbb{N}$ and $P \in \mathcal{P}$. There exists a sequence $\left(A^{n}\right)$ in $\mathcal{A}$ such that for every $n$, it holds $m^{n}+Y^{n}-X=A^{n}$. Since $\mathcal{A}^{-}$is $\mathcal{P}$-uniformly integrable, $\left(A^{n}\right)^{-}$is bounded in $L^{1}(\mathcal{P})$ and $E_{P}\left[\left(A^{n}\right)^{+}\right]=E_{P}\left[A^{n}\right]+$ $E_{P}\left[\left(A^{n}\right)^{-}\right] \leq E_{P}\left[m^{n}+Y^{n}+X^{-}\right]+E_{P}\left[\left(A^{n}\right)^{-}\right] \leq m^{n}+E_{P}\left[X^{-}\right]+E_{P}\left[\left(A^{n}\right)^{-}\right]$. This shows that $\left(A^{n}\right)$ is bounded in $L^{1}(\mathcal{P})$. Let $t \in[0, T]$ and put

$$
Y_{t}:=\liminf _{n \rightarrow \infty} M_{t}^{Z^{n}} .
$$

The process $Y$ is $\mathbb{F}$-progressively measurable and does not depend on a particular measure $P \in \mathcal{P}$. Since $\int_{0}^{t} Z_{s}^{n} d S_{s} \geq-c$, it holds $Y_{t} \geq-c$. On the other hand, it follows from Fatou's lemma and the $P$-supermartingale property of $\int Z^{n} d S$ that

$$
\begin{aligned}
E_{P}\left[Y_{t}^{+}\right] \leq \liminf _{n \rightarrow \infty} E_{P}\left[\left(\int_{0}^{t} Z_{u}^{n} d S_{u}\right)^{+}\right] \\
=\liminf _{n \rightarrow \infty}\left\{E_{P}\left[\int_{0}^{t} Z_{u}^{n} d S_{u}\right]+E_{P}\left[\left(\int_{0}^{t} Z_{u}^{n} d S_{u}\right)^{-}\right]\right\} \leq c
\end{aligned}
$$

That is, $Y_{t} \in L^{1}(P)$ and the process $Y$ is a $P$-supermartingale since for all $0 \leq s \leq$ $t \leq T$ we have $Y_{s}=\liminf _{n \rightarrow \infty} M_{s}^{Z^{n}} \geq \liminf _{n \rightarrow \infty} E_{P}\left[M_{t}^{Z^{n}} \mid \mathcal{F}_{s}\right] \geq E_{P}\left[Y_{t} \mid \mathcal{F}_{s}\right]$ Let

$$
\bar{Y}_{t}:=\limsup _{s \downarrow t, s \in \mathbb{Q} \cap[0, T]} Y_{S} \quad \text { for } t \in[0, T) \text { and } \bar{Y}_{T}:=Y_{T} .
$$

Since the filtration $\left(\mathcal{F}_{t}^{P}\right)_{t \in[0, T]}$ is right continuous for each $P \in \mathcal{P}$, the process $\bar{Y}$ is a càdlàg $P$-supermartingale with respect to $\left(\mathcal{F}_{t}^{P}\right)_{t \in[0, T]}$, see (Dellacherie and Meyer 1982, Theorems VI.2 and VI.3). Hence, $\bar{Y}$ is a $P$-supermartingale with respect 
to the filtration $\mathbb{F}$ for all $P \in \mathcal{P}$. Due to (Soner et al. 2011b, Theorem 6.5 and Proposition 6.6), there exists a $\mathbb{F}$-progressively measurable process $\bar{Z}$ and an increasing progressively measurable process $\bar{L}$ such that $\bar{L}_{0}=0$ and $\bar{Y}_{t}=\bar{Y}_{0}+\int_{0}^{t} \bar{Z}_{u} d S_{u}-\bar{L}_{t}$, where $\int \bar{Z} d S$ is a $\mathcal{P}$-local martingale. Thus, $\int_{0}^{t} \bar{Z}_{u} d S_{u} \geq \bar{Y}_{t}-\bar{Y}_{0} \geq-c-\bar{Y}_{0}$ and, by (Dellacherie and Meyer 1982, Theorem VI.2) and the right-continuity of our filtration, it holds $\bar{Y}_{0} \leq 0$ so that $M_{T}^{\bar{Z}} \in G^{c}$. Since $m^{n}+Y^{n}-X \in \mathcal{A}$ for all $n \in \mathbb{N}$ and $\mathcal{A}$ is monotone, one has $\psi^{c}(X)+\bar{Y}_{T}-X \in \mathcal{A}$ and by $\psi^{c}(X)+\int_{0}^{T} \bar{Z}_{u} d S_{u}-X \geq$ $\psi^{c}(X)+\bar{Y}_{T}-X$, it holds $\psi^{c}(X)+\int_{0}^{T} \bar{Z}_{u} d S_{u}-X \in \mathcal{A}$.

(ii) Representation: First notice that there are compact subsets $\left(K_{n}\right)$ of $\Omega$ (equipped with the maximum norm $\|\cdot\|_{\infty}$ ) such that $\Omega=\cup_{n \in \mathbb{N}} K_{n} \mathcal{P}$-q.s. To see this, let $P \in \mathcal{P}$ and $a \in A$ such that $P=P_{0} \circ\left(Y^{a}\right)^{-1}$, with $d Y_{t}^{a}=$ $a^{1 / 2}\left(Y^{a}\right) d S_{t} P_{0}$-a.s. Since $a$ is bounded, for every $q>4$ (independent of $P$ ), it holds $E_{P}\left[\left(\int_{0}^{T}\left|a_{S}\right|^{2} d\langle S\rangle_{s}\right)^{q / 4}\right]<\infty$. Thus, it follows by Burkholder-Davis-Gundy and Cauchy-Schwarz inequalities that

$$
\begin{aligned}
E_{P}\left[\left|Y_{t}^{a}-Y_{s}^{a}\right|^{q}\right] & =E\left[\left|\int_{s}^{t} a^{1 / 2}\left(Y_{.}^{a}\right) d S_{u}\right|^{q}\right] \leq C E_{P}\left[\left(\int_{s}^{t}\left|a\left(Y_{.}^{a}\right)\right| d\langle S\rangle_{u}\right)^{q / 2}\right] \\
& \leq C E_{P}\left[\left(\int_{s}^{t}\left|a\left(Y_{.}^{a}\right)\right|^{2} d\langle S\rangle_{u}\right)^{q / 4}\right]|s-t|^{q / 4} \leq K|s-t|^{q / 4}
\end{aligned}
$$

for some constants $C, K \geq 0$. Then, by (Bartl et al. 2019, Theorem A.1), $Y^{a} \in \Omega^{\alpha}$ for every $\alpha \in(0,1 / 4-1 / q)$, where $\Omega^{\alpha}$ is the space of functions $\omega \in \Omega$ which are $\alpha$ Hölder continuous. In particular, $\alpha$ can be chosen independent of $P$. Thus, $\Omega=\Omega^{\alpha}$ $\mathcal{P}$-q.s. By (Bartl et al. 2019, Corollary 3.2), $\Omega^{\alpha}=\cup_{n \in \mathbb{N}} K_{n}$ for some compact sets $K_{n}$.

Since $0 \in \mathcal{A}$, for every $X \in L^{\infty}(\mathcal{P})$, one has $\psi^{c}(X)<\infty$ and, by $\psi(0)>-\infty$, it holds $\psi^{c}(0) \in \mathbb{R}$. Thus, the convex increasing function $\psi^{c}$ is real-valued on $L^{\infty}(\mathcal{P})$. Let $\left(X^{n}\right)$ be an increasing sequence of bounded measurable functions such that $X^{n} \uparrow$ $X$. By the first part of the proof, for every $n \in \mathbb{N}$ there exists $\bar{Y}^{n} \in G^{c}$ such that $\psi^{c}\left(X^{n}\right)+\bar{Y}^{n}-X^{n} \in \mathcal{A}$ with $\bar{Y}^{n}=\int_{0}^{T} \bar{Z}_{u}^{n} d S_{u}$. Putting $Y_{t}:=\liminf _{n \rightarrow \infty} \int_{0}^{t} \bar{Z}_{u}^{n} d S_{u}$, $t \in[0, T] ; \bar{Y}_{t}:=\limsup _{s \downarrow t, s \in \mathbb{Q} \cap[0, T]} Y_{s}$ for $t \in[0, T)$; and $\bar{Y}_{T}:=Y_{T}$, we use the procedure of part (i) to construct an $S$-integrable process $\bar{Z}$ such that $\int_{0}^{t} \bar{Z}_{u} d S_{u} \geq-c$ and $\bar{Y} \leq \int \bar{Z} d S$.

Let $n \in \mathbb{N}$ be fixed. Since the function $\psi^{c}$ is increasing and the acceptance set $\mathcal{A}$ is monotone, we can find $A^{n} \in \mathcal{A}$ satisfying

$$
\left(\lim _{k \rightarrow \infty} \psi^{c}\left(X^{k}\right)\right)+\bar{Y}^{n}-X^{n}=A^{n} .
$$

Arguing as above, $\left(A^{n}\right)$ is bounded in $L^{1}(\mathcal{P})$. Hence, 


$$
\lim _{n \rightarrow \infty} \psi^{c}\left(X^{n}\right)+\int_{0}^{T} \bar{Z}_{u} d S_{u}-X \geq \lim _{n \rightarrow \infty} \psi^{c}\left(X^{n}\right)+\bar{Y}_{T}-X \geq \liminf _{n \rightarrow \infty} A^{n} \in \mathcal{A},
$$

which implies $\lim _{n \rightarrow \infty} \psi^{c}\left(X^{n}\right) \geq \psi^{c}(X)$ and therefore $\lim _{n \rightarrow \infty} \psi^{c}\left(X^{n}\right)=\psi^{c}(X)$. Thus, by (Cheridito et al. 2015, Theorem 1.7), (see also (Tangpi 2015, Theorem 4.5.2) for the probabilistic version of this result) it holds that

$$
\begin{aligned}
& \psi^{c}(X)=\sup _{Q \in c a_{1}^{+}(\tilde{\Omega})}\left(E_{Q}[X]-\psi^{c, *}(Q)\right), \quad X \in C_{b}(\mathcal{P}) \\
& \psi^{c}(X) \leq \sup _{Q \in c a_{1}^{+}(\tilde{\Omega})}\left(E_{Q}[X]-\psi^{c, *}(Q)\right), \quad X \in U_{b}(\mathcal{P}) .
\end{aligned}
$$

Notice that (Cheridito et al. 2015, Theorem 1.7) (respectively, (Tangpi 2015, Theorem 4.5.2)) require $\Omega$ to be $\sigma$-compact, (respectively, be quasi-surely equal to a $\sigma$-compact set). The property $\psi^{c, *}(Q)=\phi^{c, *}(Q)+\rho_{\mathcal{A}}^{*}(-Q)$, for all $Q \in c a_{1}^{+}(\tilde{\Omega})$ is a consequence of Lemma 1 . Thus, if $\rho_{\mathcal{A}}^{*}(-Q)=\infty$, then $\psi^{c, *}(Q)=\infty$, so that (13) and (14) can be deduced from (15) and (16), respectively.

Proof (of Theorem 1). Since $G^{n} \subseteq G$ for all $n \in \mathbb{N} \backslash\{0\}$, one has $\psi(X) \leq$ $\inf _{n \geq 1} \psi^{n}(X)$. Assume that the inequality is strict, that is, $\psi(X)<\inf _{n \geq 1} \psi^{n}(X)$. Then, there are $m \in \mathbb{R}$ and $\varepsilon>0$ such that $\psi(X)<m<m+\varepsilon<\inf _{n \geq 1} \psi^{n}(X)$. Thus, there is $x \in \mathbb{R}$ such that $\psi(X) \geq x-\varepsilon$, with $x+Y-X \in \mathcal{A}$ for some $Y \in G$. Since there is $n \in \mathbb{N}$ such that $Y \in G^{n}$, we have $x \geq \psi^{n}(X)$. Hence,

$$
m>\psi(X) \geq x-\varepsilon \geq \psi^{n}(X)-\varepsilon \geq \inf _{n \geq 1} \psi^{n}(X)-\varepsilon,
$$

which is a contradiction. Thus, $\psi(X)=\inf _{n \geq 1} \psi^{n}(X)$, the sequence $\left(\psi^{n, *}(Q)\right)_{n}$ is increasing and $\psi^{*}(Q)=\sup _{n \geq 1} \psi^{n, *}(Q)$ for all $Q \in c a_{1}^{+}(\tilde{\Omega})$.

Let $X \in C_{b}(\mathcal{P})$. By Proposition 1 , for every $n \geq 1$ it holds $\psi(X) \leq$ $\sup _{Q \in \mathcal{M}_{\mathcal{P}}^{\mathcal{A}}}\left(E_{Q}[X]-\psi^{n, *}(Q)\right)$. Thus, there is $Q^{n} \in c a_{1}^{+}(\overline{\tilde{\Omega}})$ such that

$$
\psi(X) \leq E_{Q^{n}}[X]-\psi^{n, *}\left(Q^{n}\right)+\frac{1}{n} .
$$

Since $X$ is bounded and $\rho_{\mathcal{A}}^{*}(-Q) \leq \psi^{n, *}(Q)$, there is a constant $c \geq 0$ such that $Q^{n} \in\left\{Q \in c a_{1}^{+}(\tilde{\Omega}): \rho_{\mathcal{A}}^{*}(-Q) \leq c\right\}$ for all $n$. Hence, there is $Q \in c a_{1}^{+}$such that up to a subsequence, $\left(Q^{n}\right)$ converges to $Q$ in $\sigma\left(c a_{1}^{+}, C_{b}\right)$ and since $\tilde{\Omega}$ is closed, we have $1=\limsup _{n \rightarrow \infty} Q^{n}(\tilde{\Omega}) \leq Q(\tilde{\Omega})$, showing that actually, $Q \in c a_{1}^{+}(\tilde{\Omega})$. Now, let $\varepsilon>0$ and $N \in \mathbb{N}$ be such that $\psi^{N, *}(Q) \geq \psi^{*}(Q)-\varepsilon$. Since $\psi^{N \text {,* }}$ is lower semicontinuous (with $c a_{1}^{+}$equipped with the weak topology $\sigma\left(c a_{1}^{+}, C_{b}\right)$ ), we can choose $n \geq N$ large enough so that $\psi^{N, *}\left(Q^{n}\right) \geq \psi^{N, *}(Q)-\varepsilon$. Thus,

$$
\psi^{n, *}\left(Q^{n}\right) \geq \psi^{N, *}\left(Q^{n}\right) \geq \psi^{N, *}(Q)-\varepsilon \geq \psi^{*}(Q)-2 \varepsilon .
$$

This shows that $\psi(X) \leq E_{Q^{n}}[X]-\psi^{*}(Q)-2 \varepsilon+1 / n$. Taking the limit in $n$ and dropping $\varepsilon$ yields

$$
\psi(X) \leq E_{Q}[X]-\psi^{*}(Q)
$$


Since the weak duality $\psi(X) \geq \sup _{Q \in c a_{1}^{+}(\tilde{\Omega})}\left(E_{Q}[X]-\psi^{*}(Q)\right), X \in C_{b}(\mathcal{P})$ is easily obtained, this implies

$$
\psi(X)=\sup _{Q \in c a_{1}^{+}(\tilde{\Omega})}\left(E_{Q}[X]-\psi^{*}(Q)\right) \quad X \in C_{b}(\mathcal{P}) .
$$

Let us now show that $\psi^{*}(Q)=\infty$ whenever $Q \notin \mathcal{M}_{\mathcal{P}}^{\mathcal{A}}(S)$. Since $0 \in G$, we have $\psi(X) \leq \rho_{\mathcal{A}}(-X)$ for every $X \in C_{b}$, and hence $\psi^{*}(Q) \geq \rho_{\mathcal{A}}^{*}(-Q)$ for every $Q \in c a_{1}^{+}(\tilde{\Omega})$. Thus, if $\rho_{\mathcal{A}}^{*}(-Q)=\infty$, then $\psi^{*}(Q)=\infty$. If $S$ is not a $Q$-local martingale, then since $\operatorname{supp}(Q) \subseteq \tilde{\Omega}$ and $\tilde{\Omega}$ is a subset of a $\sigma$-compact set, it follows from (Bartl et al. 2019, Remark 4.1 and Proposition 4.4) that there is $X \in C_{b}$ and an $S$-integrable process $Z$ such that $X \leq M^{Z}$ and $E_{Q}[X]>0$. Thus, one has $\psi(x X) \leq 0$ for all $x \geq 0$ and by $X \in C_{b}$ it holds $\psi^{*}(Q) \geq E_{Q}[x X]$ for all $x \geq 0$. This shows by scaling that $\psi^{*}(Q)=\infty$. It remains to show that $\phi^{*}(Q)=0$ whenever $S$ is a $Q$-local martingale. For every bounded $X$ and every $\varepsilon>0$, there is $m \in \mathbb{R}$ such that $\phi(X) \geq x-\varepsilon$ and $x+M_{T}^{Z} \geq X$ for some admissible $Z$. Since $M^{Z}$ is a $Q$-local martingale that is bounded from below, it is a $Q$-supermartingale. Thus, $E_{Q}[X]-\phi(X)-\varepsilon \leq E_{Q}[X]-x \leq 0$. Since $\varepsilon$ was taken arbitrarily, this shows that $\phi^{*}(Q) \leq 0$, and since we also have $\phi^{*}(Q) \geq 0$, the result is obtained. Arguing as in the proof of Proposition 1, we have $\psi^{*}(Q)=\phi^{*}(Q)+\rho_{\mathcal{A}}^{*}(-Q)$. Therefore, (6) is due to (17).

Furthermore, it follows again by Proposition 1 that

$$
\psi^{n}(X) \leq \sup _{Q \in \mathcal{M}_{\mathcal{P}}^{\mathcal{A}}}\left(E_{Q}[X]-\psi^{n, *}(Q)\right), \quad X \in U_{b}(\mathcal{P}) .
$$

Let $X \in U_{b}(\mathcal{P})$. For every $n \geq 1$, it holds $\psi(X) \leq \sup _{Q \in \mathcal{M}_{\mathcal{P}}^{\mathcal{A}}}\left(E_{Q}[X]-\psi^{n, *}(Q)\right)$. Arguing as above, we find $Q^{n}, Q \in c a_{1}^{+}(\tilde{\Omega})$ such that $\left(Q^{n}\right)$ converges to $Q$ in $\sigma\left(c a_{1}^{+}, C_{b}\right)$, and for every $\varepsilon>0$, up to a subsequence, $\psi(X) \leq E_{Q^{n}}[X]-\psi^{*}(Q)-$ $2 \varepsilon+1 / n$. Since $X$ is upper semicontinuous and bounded, taking the limit this implies $\psi(X) \leq E_{Q}[X]-\psi^{*}(Q)$, showing that

$$
\psi(X) \leq \sup _{Q \in \mathcal{M}_{\mathcal{P}}^{A}}\left(E_{Q}[X]-\psi^{*}(Q)\right), \quad X \in U_{b}(\mathcal{P}) .
$$

The assumption $\psi^{*}(Q)=\sup _{X \in U_{b}}\left(E_{Q}[X]-\psi(X)\right)$ implies that the inequality in (18) is an equality and as shown above, $\psi^{*}(Q)=\infty$ whenever $Q \notin \mathcal{M}_{\mathcal{P}}^{\mathcal{A}}(S)$. This concludes the proof.

\subsection{Proof of theorem 2}

Recall that here, $\mathcal{A}$ is given by

$$
\mathcal{A}:=\left\{X \in L^{1}(\mathcal{P}): \rho(X) \leq 0\right\},
$$

the acceptance set of the robust optimized certainty equivalent defined by (8). 
Lemma 2 If there exist $a>0, b \in \mathbb{R}$, and $p>2$ such that the loss function $l$ satisfies the growth condition $l(x) \geq a|x|^{p}+b$, then the set $\mathcal{A}^{-}:=\left\{A^{-}: A \in \mathcal{A}\right\}$ is $\mathcal{P}$-uniformly integrable.

Proof Consider the classical OCE $\rho^{P}$ defined as

$$
\rho^{P}(X):=\inf _{m \in \mathbb{R}}\left(E_{P}[l(m-X)]-m\right) .
$$

By definition, we have

$$
\rho(X) \geq \sup _{P \in \mathcal{P}} \inf _{m \in \mathbb{R}}\left(E_{P}[l(m-X)]-m\right)=\sup _{P \in \mathcal{P}} \rho^{P}(X) .
$$

Assume by contradiction that there is $\bar{P} \in \mathcal{P}$ and $\varepsilon>0$ such that it holds

$$
\liminf _{n \rightarrow \infty} \sup _{X \in \mathcal{A}} E_{\bar{P}}\left[X^{-} 1_{\left\{X^{-} \geq n\right\}}\right] \geq \varepsilon .
$$

Given $X \in \mathcal{A}$, put $\delta^{n}:=E_{\bar{P}}\left[X^{-} 1_{\left\{X^{-} \geq n\right\}}\right] \geq \varepsilon$. Let $Q^{n} \ll \bar{P}$ be the measure given by $\frac{d Q^{n}}{d \bar{P}}:=X^{-} 1_{\left\{X^{-} \geq n\right\}} / \delta^{n}$.

Notice that $l^{*}$ satisfies the growth condition $l^{*}(z) \leq a^{\prime}|z|^{q}+b$ with the constant $a^{\prime}=\frac{1-a}{(a p)^{q}}$ and where $1<q<2$ is the Hölder conjugate of $p$. Thus, it holds that

$$
\begin{aligned}
\rho(X) \geq \rho^{\bar{P}}(X) & \geq E_{\bar{P}}\left[-X \frac{X^{-} 1_{\left\{X^{-} \geq n\right\}}}{\delta_{n}}\right]-E_{\bar{P}}\left[l^{*}\left(\frac{X^{-} 1_{\left\{X^{-} \geq n\right\}}}{\delta^{n}}\right)\right] \\
& \geq E_{\bar{P}}\left[\left(X^{-}\right)^{2} 1_{\left\{X^{-} \geq n\right\}}\right] \frac{1}{\delta^{n}}-a^{\prime} E_{\bar{P}}\left[\left(X^{-}\right)^{q} 1_{\left\{X^{-} \geq n\right\}}\right] \frac{1}{\left(\delta^{n}\right)^{q}}-b \\
& \geq E_{\bar{P}}\left[\left(X^{-}\right)^{q} 1_{\left\{X^{-} \geq n\right\}}\right]\left(\frac{n^{2-q}}{\delta^{n}}-\frac{a^{\prime}}{\left(\delta^{n}\right)^{q}}\right)-b \\
& \geq\left(E_{\bar{P}}\left[X^{-} 1_{\left\{X^{-} \geq n\right\}}\right]\right)^{q}\left(\frac{n^{2-q}}{\delta^{n}}-\frac{a^{\prime}}{\left(\delta^{n}\right)^{q}}\right)-b,
\end{aligned}
$$

where the last inequality follows by Jensen's inequality with $n$ large enough. Since $q<2$, the last term converges to infinity, a contradiction.

Observe that our verification of the uniform integrability property of $\mathcal{A}^{-}$in the case of the optimized certainty equivalent (see the preceding proof) requires the loss function $l$ to grow sufficiently fast. This is why Theorem 2 does not apply, say to the average value at risk which is the OCE risk measure with loss function $l(x):=x^{+}$.

Recall the conjugate function $\rho^{*}$ defined in section 2.2 as

$$
\rho^{*}(-Q):=\sup _{X \in C_{b}}\left(E_{Q}[-X]-\rho(X)\right) \text {. }
$$

Lemma 3 If $\mathcal{P}$ is $\sigma\left(c a_{1}^{+}, C_{b}\right)$-compact, then it holds

$$
\rho^{*}(-Q)=\inf _{P \in \mathcal{P}} E_{P}\left[l^{*}\left(\frac{d Q}{d P}\right)\right] \text { for all } Q \in c a_{1}^{+}
$$

and the sublevel sets $\left\{Q \in c a_{1}^{+}: \rho^{*}(-Q) \leq c\right\}, c \geq 0$ are $\sigma\left(c a_{1}^{+}, C_{b}\right)$-compact. 
Proof Let us first prove (20). Since for each $X \in C_{b}$ the function $P \mapsto \rho^{P}(X)$ is concave and $\sigma\left(c a_{1}^{+}, C_{b}\right)$-upper semicontinuous, it follows by weak compactness of $\mathcal{P}$ and (Fan (1953), Theorem 2) that

$$
\rho^{*}(-Q)=\inf _{P \in \mathcal{P}} \sup _{X \in C_{b}}\left(E_{Q}[-X]-\rho^{P}(X)\right) .
$$

Let $L^{\infty}\left(P, \mathcal{F}_{T}^{S}\right)$ be the space of $P$-essentially bounded and $\mathcal{F}_{T}^{S}$-measurable random variables. We claim that

$$
\sup _{X \in C_{b}}\left(E_{Q}[-X]-\rho^{P}(X)\right)=\sup _{X \in L^{\infty}\left(P, \mathcal{F}_{T}^{S}\right)}\left(E_{Q}[-X]-\rho^{P}(X)\right)=E_{P}\left[l^{*}(d Q / d P)\right]
$$

for every Borel measure $Q \ll P$. The second equality of the claim follows by (BenTal and Taboulle 2007). To prove the first one, let $\varepsilon>0$ and $X \in L^{\infty}\left(P, \mathcal{F}_{T}^{S}\right)$ be such that

$$
\sup _{X \in L^{\infty}\left(P, \mathcal{F}_{T}^{S}\right)}\left(E_{Q}[-X]-\rho^{P}(X)\right) \leq E_{Q}[-X]-\rho^{P}(X)+\varepsilon .
$$

It follows by Lusin's and Tietze's theorems that there is a sequence $\left(X^{n}\right)$ of continuous functions converging $P$-a.s. to $X$, see, for instance, (Wisniewski 1994, Theorem 1) for details. In addition, the sequence $\left(X^{n}\right)$ can be chosen bounded. Since $l^{*}(x) / x \rightarrow+\infty$ as $|x|$ goes to infinity, it follows that for each $c \geq 0$, the set $\left\{d Q / d P: E_{P}\left[l^{*}(d Q / d P)\right] \leq c\right\}$ is $\sigma\left(L^{1}\left(P, \mathcal{F}_{T}^{S}\right), L^{\infty}\left(P, \mathcal{F}_{T}^{S}\right)\right)$-compact. Hence, by the representation

$$
\rho^{P}(X)=\sup _{Q \ll P}\left(E_{Q}[-X]-E_{P}\left[l^{*}(d Q / d P)\right]\right) \quad X \in L^{\infty}\left(P, \mathcal{F}_{T}^{S}\right),
$$

see, for instance, (Ben-Tal and Taboulle 2007, Theorem 4.2), and the JouiniSchachermayer-Touzi theorem (see (Jouini et al. 2006, Theorem 2.4)), one has $\lim _{n \rightarrow \infty} \rho^{P}\left(X^{n}\right)=\rho^{P}(X)$. Therefore, $E_{Q}[-X]-\rho^{P}(X)=\lim _{n \rightarrow \infty} E_{Q}\left[-X^{n}\right]-$ $\rho^{P}\left(X^{n}\right)$, which proves the claim. In combination with (21), we obtain (20).

Next, let us prove compactness of the sublevel sets. As a consequence of Prokhorov's theorem, the set $\mathcal{P}$ is tight. That is, there exists a family $\left(K_{n}\right)$ of compact subsets of $\Omega$ such that $\sup _{P \in \mathcal{P}} P\left(K_{n}^{c}\right) \rightarrow 0$. Let $Q \in c a_{+}^{1}$ satisfy $\rho^{*}(-Q) \leq C$. There is $P \in \mathcal{P}$ such that $Q \ll P$ and $E_{P}\left[l^{*}\left(\frac{d Q}{d P}\right)\right] \leq C+1$. Therefore for all $m>0$, by Young's inequality, one has

$$
m \frac{d Q}{d P} 1_{K_{n}^{c}} \leq l\left(m 1_{K_{n}^{c}}\right)+l^{*}\left(\frac{d Q}{d P}\right)
$$

and using $l(0)=0$, one gets

$$
Q\left(K_{n}^{c}\right) \leq \frac{1}{m}\left(l(m) P\left(K_{n}^{c}\right)+E_{P}\left[l^{*}\left(\frac{d Q}{d P}\right)\right]\right) \leq \frac{l(m)}{m} \sup _{P \in \mathcal{P}} P\left(K_{n}^{c}\right)+\frac{(C+1)}{m} .
$$

Thus, $\sup _{\left\{Q: \rho^{*}(-Q) \leq C\right\}} Q\left(K_{n}^{c}\right) \rightarrow 0$ as $n \rightarrow \infty$, showing by Prokhorov's theorem that the sublevel set is weakly relatively compact. Since $\rho^{*}$ is lower semicontinuous, the sublevel sets are $\sigma\left(c a_{1}^{+}, C_{b}\right)$-closed. This completes the argument. 
Lemma 4 For every local martingale measure $Q$ of $S$ such that $Q \ll P$ for some $P \in \mathcal{P}$, and every $Y \in G$, it holds $E_{Q}[Y] \leq 0$.

Remark 2 Note that in the above lemma, $Y \in G$ is not necessarily a $Q$-stochastic integral.

Proof (of Lemma 4). Let $Y \in G$ and $Q$ be a local martingale measure for $S$. Let $P \in \mathcal{P}$ be such that $Q \ll P$, let $c>0$, and $Z$ an $S$-integrable process such that $Y_{t}:=\int_{0}^{t} Z_{u} d S_{u} \geq-c$. Recall that a process $H:[0, T] \times \Omega \rightarrow \mathbb{R}$ is called simple if it is of the form

$$
H_{t}(\omega)=\sum_{i=1}^{N} h_{i}(\omega) 1_{\left(\tau_{i}(\omega), \tau_{i+1}(\omega)\right]},
$$

where $N \in \mathbb{N}, 0 \leq \tau_{1} \leq \cdots \leq \tau_{N+1} \leq T$ are $\mathbb{F}$-stopping times and $h_{i}$ are $\mathcal{F}_{\tau_{i}}{ }^{-}$ measurable bounded functions.

Let us first assume that $E_{P}\left[\int_{0}^{T}\left|Z_{u}\right|^{2} d u\right]<\infty$. Then, there is a sequence $\left(Z^{n}\right)$ of simple processes such that $\int_{0}^{T} Z_{u}^{n} d S_{u} \rightarrow \int_{0}^{T} Z_{u} d S_{u}$ in $L^{2}(P)$. Fix $\varepsilon>0$, and define the sequence of stopping times

$$
\tau^{n}:=\inf \left\{t>0: \int_{0}^{t} Z_{u}^{n} d S_{u} \leq-c-\varepsilon\right\} \wedge T,
$$

with the convention inf $\emptyset:=+\infty$. Further, put $\tilde{Z}^{n}:=Z^{n} 1_{\left[0, \tau^{n}\right]}$. By definition, $\int_{0}^{t} \tilde{Z}_{u}^{n} d S_{u}=\int_{0}^{t \wedge \tau^{n}} Z_{u}^{n} d S_{u} \geq-c-\varepsilon$. For almost all $\omega \in \Omega$, there is $N \in \mathbb{N}$ such that if $n \geq N$, then $\int_{0}^{t} Z_{u}^{n} d S_{u}(\omega) \geq \int_{0}^{t} Z_{u} d S_{u}(\omega)-\varepsilon \geq-c-\varepsilon$. Thus, $\tau^{n}(\omega)=T$, in other words, $\tau^{n} \uparrow T P$-a.s. Hence, $\int_{0}^{T} \tilde{Z}_{u}^{n} d S_{u}=\int_{0}^{\tau^{n}} Z_{u}^{n} d S_{u}$ converges to $\int_{0}^{T} Z_{u} d S_{u}$ $P$-a.s. and $Q$-a.s. In addition, since $\tilde{Z}^{n}$ is a simple process, $\int \tilde{Z}^{n} d S$ is a $Q$-martingale, so that $E_{Q}\left[\int_{0}^{T} \tilde{Z}_{u}^{n} d S_{u}\right]=0$. Therefore, it follows from Fatou's lemma that

$$
0=\liminf _{n \rightarrow \infty} E_{Q}\left[\int_{0}^{T} \tilde{Z}_{u}^{n} d S_{u}\right] \geq E_{Q}\left[\int_{0}^{T} Z_{u} d S_{u}\right] .
$$

In the general case, let $\sigma^{k}$ be a localizing sequence such that $\int_{0}^{\sigma^{k} \wedge} Z d S$ is a square integrable $P$-martingale. Put $Z^{k}:=Z 1_{\left[0, \sigma^{k}\right]}$. One has $\int_{0}^{t} Z_{u}^{k} d S_{u} \geq-c$ for all $k$. By the first part of the proof, for each $k$, it holds $E_{Q}\left[\int_{0}^{T} Z_{u}^{k} d S_{u}\right] \leq 0$. Taking the limit in $k$, it follows by Fatou's lemma that $E_{Q}\left[\int_{0}^{T} Z_{u} d S_{u}\right] \leq 0$.

Proof (of Theorem 2). It is clear that the set $\mathcal{A}$ contains 0 , and is convex and monotone. Moreover, in view of Theorem 1, Lemmas 2 and 3, the representation can be obtained if we show that $X:=\liminf _{n \rightarrow \infty} X^{n} \in \mathcal{A}$ for every sequence $\left(X^{n}\right)$ in $\mathcal{A}$ that is bounded in $L^{1}(\mathcal{P})$ and that

$$
\psi^{*}(Q)=\psi_{U_{b}}^{*}(Q):=\sup _{X \in U_{b}}\left(E_{Q}[X]-\psi(X)\right) .
$$

For every $n \in \mathbb{N}$, there is an $m^{n} \in \mathbb{R}$ such that

$$
E_{P}\left[l\left(m^{n}-X^{n}\right)\right]-m^{n}-1 / n \leq 0 \text { for all } P \in \mathcal{P} .
$$


Condition (CIB) ensures that $l(x) \geq b x+c$ and $l(x) \geq b^{\prime} x+c$ for all $x \in \mathbb{R}$ for some $b>1>b^{\prime}$ and $c \in \mathbb{R}$. Since $\left(X^{n}\right)$ is bounded in $L^{1}(\mathcal{P})$, this shows that $\left(m^{n}\right)$ is bounded. Thus, there is an $m$ such that $\left(m^{n}\right)$ converges to $m$ after passing to a subsequence. Hence, it follows from Fatou's lemma and the continuity of $l$ that $E_{P}[l(m-X)]-m \leq 0$. Since this holds for every $P \in \mathcal{P}$, it follows that $\rho(X) \leq 0$, from which we conclude that $X \in \mathcal{A}$.

Let us now prove (22). It follows from Theorem 1 and Lemma 3 that

$$
\psi^{*}(Q)=\phi^{*}(Q)+\inf _{P \in \mathcal{P}} E_{P}\left[l^{*}(d Q / d P)\right]
$$

Observe that by definition, $\psi^{*} \leq \psi_{U_{b}}^{*}$. Let $Q \in c a_{1}^{+}(\tilde{\Omega})$. Assuming that $\inf _{P \in \mathcal{P}} E_{P}\left[l^{*}(d Q / d P)\right]=\infty$, then by Lemma $3, \psi^{*}(Q) \geq \rho^{*}(-Q)=\infty$. If $S$ is not a $Q$-local martingale, then $\operatorname{since} \operatorname{supp}(Q) \subseteq \tilde{\Omega}$ and $\tilde{\Omega}$ is a subset of a $\sigma$-compact set, it follows from (Bartl et al. 2019, Remark 4.1 and Proposition 4.4) that there is $X \in C_{b}$ and an $S$-integrable process $Z$ such that $X \leq M^{Z}$ and $E_{Q}[X]>0$. Thus, one has $\phi(x X) \leq 0$ for all $x \geq 0$ and by $X \in C_{b}$ it holds that $\psi^{*}(Q) \geq \phi^{*}(Q) \geq$ $E_{Q}[x X]$ for all $x \geq 0$. This shows by scaling that $\psi^{*}(Q)=\phi^{*}(Q)=\infty$. Thus, $\infty=\psi^{*}(Q) \leq \psi_{U_{b}}^{*}(Q)$ for all $Q \notin \mathcal{M}_{\mathcal{P}}^{l}(S)$.

On the other hand, it can be checked that $\rho$ satisfies the weak duality

$$
\rho(X) \geq \sup _{Q}\left(E_{Q}[-X]-\inf _{P \in \mathcal{P}} E_{P}\left[l^{*}(d Q / d P)\right]\right) \quad \text { for all } X \in L^{1}(\mathcal{P}) .
$$

Let $Q \in \mathcal{M}_{\mathcal{P}}^{l}(S)$, and $X \in U_{b}$ such that $m+Y-X \in \mathcal{A}$ for some $m \in \mathbb{R}$ and $Y \in G$. It holds that $E_{Q}[-m-Y+X]-\rho^{*}(-Q) \leq 0$ and there is $P \in \mathcal{P}$ such that $Q \ll P$. By Lemma 4 , we have $E_{Q}[Y] \leq 0$, that is, $E_{Q}[X]-m \leq \rho^{*}(-Q)$. This implies $\psi_{U_{b}}^{*}(Q) \leq \rho^{*}(-Q) \leq \psi^{*}(Q)$. Therefore, $\psi^{*}=\psi_{U_{b}}^{*}$.

Finally, recall that $\psi^{*}(Q)=\phi^{*}(Q)+\rho^{*}(-Q)$ for every $Q$ and $\phi^{*}(Q)=0$ for $Q \in \mathcal{M}_{\mathcal{P}}^{l}(S)$. This concludes the proof.

\section{Appendix A: Separable class of diffusion coefficients}

In this appendix, we define the classes of diffusion coefficients we consider. The definition and the proposition below are taken from Soner et al. (2011b). Let $\overline{\mathcal{P}}_{W}$ be the set of local martingale measures $P$ of $S$ such that $P$-a.s., $\langle S\rangle_{t}$ is absolutely continuous in $t$ and $\hat{a}$ takes values in $\mathbb{S}_{d}^{>0}$, put

$$
\bar{A}:=\left\{a: \mathbb{R}_{+} \rightarrow \mathbb{S}_{d}^{>0}, \mathbb{F} \text {-progressively measurable and } \int_{0}^{t}\left|a_{s}\right| d s<\infty \text { for all } t \geq 0\right\},
$$

and for each $P \in \overline{\mathcal{P}}_{W}, \bar{A}_{W}(P):=\{a \in \bar{A}: a=\hat{a} P$-a.s. $\}$. Denote by $\bar{A}_{W}$ the set $\bar{A}_{W}:=\cup_{P \in \overline{\mathcal{P}}_{W}} \bar{A}_{W}(P)$ and by $A_{W}$ the set of elements of $\bar{A}_{W}$ such that the SDE (3) has weak uniqueness.

Definition $1 A$ subset $A_{0}$ of $A_{W}$ is called a generating class of diffusion coefficients if 
(i) $A_{0}$ satisfies the concatenation property: $a 1_{[0, t)}+b 1_{[t, \infty)} \in A_{0}$ for all $a, b \in$ $A_{0}$ and $t \geq 0$.

(ii) $A_{0}$ has constant disagreement times: for all $a, b \in A_{0}, \theta^{a, b}$ is a constant, with $\theta^{a, b}: \inf \left\{t \geq 0:=\int_{0}^{t} a_{s} d s \neq \int_{0}^{t} b_{s} d s\right\}$.

Definition 2 A set $A$ is a separable class of diffusion coefficients generated by $A_{0}$ if $A_{0} \subseteq A_{W}$ is a generating class of diffusion coefficients and A consists of all processes of the form

$$
a=\sum_{n=0}^{\infty} \sum_{i=1}^{\infty} a_{i}^{n} 1_{E_{i}^{n}} 1_{\left[\tau_{n}, \tau_{n+1}\right)},
$$

where $\left(a_{i}^{n}\right)_{i, n} \subseteq A_{0},\left(\tau_{n}\right)_{n}$ is an increasing sequence of $\mathbb{F}$-stopping times valued in $\mathbb{R}_{+} \cup\{\infty\}$ with $\tau_{0}=0$ and

(i) $\inf \left\{n: \tau_{n}=\infty\right\}<\infty, \tau_{n}<\tau_{n+1}$ whenever $\tau_{n}<\infty$, and each $\tau_{n}$ takes at most countably many values.

(ii) for each $n,\left\{E_{i}^{n}, i \geq 1\right\} \subseteq \mathcal{F}_{\tau_{n}}$ forms a partition of $\Omega$.

Proposition 2 Let $A$ be a separable class of diffusion coefficients generated by $A_{0}$. Then, $A \subseteq A_{W}$ and if for all $a \in A_{0}, P^{a}$ satisfies the martingale representation property, then for all $a \in A, P^{a}$ satisfies the martingale representation property as well.

Acknowledgements Heartfelt thanks are due to Daniel Bartl for fruitful discussions. In addition the author would like to thank two anonymous referees.

\section{Authors' contributions}

The author read and approved the final manuscript.

\section{Funding}

Not applicable.

\section{Availability of data and materials}

Not applicable.

\section{Competing interests}

Not applicable.

\section{References}

Acciaio, B., Beiglböck, M., Penkner, F., Schachermayer, W.: A model-free version of the fundamental theorem of asset pricing and the super-replication theorem. Math. Finance. 26(2), 233-251 (2016)

Aliprantis, C.D., Border, K.C.:: Infinite Dimensional Analysis: a Hitchhiker's Guide, 3rd ed. Springer (2006) 
Arai, T.: Convex risk measures on orlicz spaces: inf-convolution and shortfall. Math. Finan. Econ. 3, 7388 (2010)

Bartl, D., Drapeau, S., Tangpi, L.: Computational aspects of robust optimized certainty equivalents and option pricing. Math. Finance. 30(1), 287-09 (2020)

Bartl, D., Kupper, M., Prömel, D.J., Tangpi, L.: Duality for pathwise superhedging in continuous time. Finance Stoch. 23(3), 697-728 (2019)

Bartl, D., Kupper, M., Neufeld, A.: Pathwise superhedging on prediction sets. Finance Stoch. 24, 215-48 (2020)

Becherer, D., Kentia, K.: Good deal hedging and valuation under combined uncertainty about drift and volatility. Probab. Uncertain. Quant. Risk. 2(13) (2017)

Beiglböck, M., Henry-Labordère, P., Penkner, F.: Model-independent bounds for option prices - a mass transport approach. Finance Stoch. 17(3), 477-501 (2013)

Ben-Tal, A., Taboulle, M.: An old-new concept of convex risk measures: The optimized certainty equivalent. Math. Finance. 17, 449-476 (2007)

Bion-Nadal, J., Di Nunno, G.: Dynamic no-good-deal pricing measures and extension theorems for linear operators on $L^{\infty}$. Finance Stoch. 17(3), 587-613 (2013)

Bion-Nadal, J., Kervarec, M.: Risk Measuring under Model Uncertainty. Ann. Appl. Probab. 22(1), 213238 (2012)

Burzoni, M.: Arbitrage and hedging in model independent markets with frictions. SIAM J. Financial Math. 7(1), 812-844 (2016)

Burzoni, M., Frittelli, M., Maggis, M.: Model-free superhedging duality. Ann. Appl. Probab. 27(3), 14521477 (2017)

Cheridito, P., Kupper, M., Tangpi, L.: Representation of increasing convex functionals with countably additive measures. Preprint (2015)

Cheridito, P., Kupper, M., Tangpi, L.: Duality formulas for robust pricing and hedging in discrete time. SIAM J. Financial Math. 8(1), 738-765 (2017)

Delbaen, F., Schachermayer, W.: A general version of the fundamental theorem of asset pricing. Math. Ann. 300(3), 463-520 (1994)

Dellacherie, C., Meyer, P.-A.:: Probabilities and Potential. B. North-Holland Mathematics Studies, vol. 72, p. 463. North-Holland Publishing Co., Amsterdam (1982). Theory of martingales, Translated from the French by J. P. Wilson

Denis, L., Martini, C.: A theoretical framework for the pricing of contingent claims in the presence of model uncertainty. Ann. Appl. Probab. 16, 827-852 (2006)

Dolinsky, Y., Soner, H.M.: Martingale optimal transport and robust hedging in continuous time. Probab. Theory Relat. Fields. 160(1-2), 391-427 (2014)

Fan, K.: Minimax theorems. Proc. Nat. Acad. Sci. U.S.A. 39, $42-47$ (1953)

Föllmer, H., Leukert, P.: Quantile hedging. Finance Stoch. 3(3), 251-273 (1999)

Föllmer, H., Leukert, P.: Efficient hedging: Cost versus shortfall risk. Finance Stoch. 4, 117-146 (2000)

Hou, Z., Obłój, J.: On robust pricing-hedging duality in continuous time. Finance Stoch. 22(3), 511-567 (2018)

Jouini, E., Schachermayer, W., Touzi, N.: Law invariant risk measures have the fatou property. Adv. Math. Econ. 9, 49-71 (2006)

Kaina, M., Rüschendorf, L.: On convex risk measures on $l^{p}$-spaces. Math. Meth. Oper. Res. 69(3), 475495 (2009)

Karandikar, R.L.: On quadratic variation process of a continuous martingale. Illinois J. Math. 27, 178-181 (1983)

Karatzas, I., Shreve, S.E.:: Brownian Motion and Stochastic Calculus (Graduate Texts in Mathematics). Springer, New York (2004)

Kramkov, D., Schachermayer, W.: The Asymptotic Elasticity of Utility Functions and Optimal Investment in Incomplete Market. Ann. Appl. Probab. 9(3), 904-950 (1999)

Kupper, M., Schachermayer, W.: Representation results for law invariant time consistent functions. Math. Financ. Econ. 2(3), 189-210 (2009)

Neufeld, A., Nutz, M.: Superreplication under Volatility Uncertainty for Measurable Claims. Electron. J. Probab. 18(48), 1-14 (2013)

Peng, S.: Nonlinear expectations and stochastic calculus under uncertainty. arXiv Preprint 1002.4546 (2010)

Rudloff, B.: Convex hedging in incomplete markets. Appl. Math. Finance. 14(5), 437-452 (2007) 
Soner, H.M., Touzi, N., Zhang, J.: Martingale representation theorem for the $G$-expectation. Stoch. Proc. Appl. 121(2), 265-287 (2011a)

Soner, M.H., Touzi, N., Zhang, J.: Quasi-sure stochastic analysis through aggregation. Electron. J. Probab. 16(67) (2011b)

Soner, H.M., Touzi, N., Zhang, J.: Dual formulation of second order target problems. Ann. Appl. Probab. 23(1), 308-347 (2013)

Tangpi, L.: Dual Representation of Convex Increasing Functionals with Applications to Finance. PhD thesis, University of Konstanz (2015)

Wisniewski, A.: The structure of measurable mappings on metric spaces. Proc. A.M.S. 122(1), 147-150 (1994) 